\title{
The symmetry structure of the anti-self-dual Einstein hierarchy
}

\author{
I.A.B.Strachan \\ Dept. of Mathematics and Statistics, University of Newcastle, \\ Newcastle-upon-Tyne, NE1 7RU, England @.
}

\begin{abstract}
An important example of a multi-dimensional integrable system is the anti-self-dual Einstein equations. By studying the symmetries of these equations, a recursion operator is found and the associated hierarchy constructed. Owing to the properties of the recursion operator one may construct a hierarchy of symmetries and find the algebra generated by them. In addition, the Lax pair for this hierarchy is constructed.
\end{abstract}

\section{Introduction}

One fundamental idea within the theory of integrable systems is that of a hierarchy, the paradigm being the KdV hierarchy

$$
u_{t}=\mathcal{R}^{n} u_{x}
$$

where $\mathcal{R}$ is the recursion operator, defined by $\mathcal{R}=\partial_{x}^{2}+4 u+2 u_{x} \partial_{x}^{-1}$. One of the ways of introducing this operator is via the symmetries of the original $\mathrm{KdV}$ equation, the quantities $\mathcal{R}^{n} u_{x}$ being generalized symmetries of the KdV equation. Further analysis of the recursion operator leads one to the concepts of commuting flows and the biHamiltonian structure of the KdV hierarchy.

This structure persists in higher dimensional integrable systems. The hierarchy associated with the anti-self-dual Yang-Mills equations has been studied by many authors [1, 2, 3, 4, and has all the properties one would expect from an integrable system - the existence of recursion operators and bi-Hamiltonian structure to name but two.

\footnotetext{
${ }^{1}$ e-mail: i.a.b.strachan@newcastle.ac.uk
} 
The purpose of this paper is to study the hierarchical structure of another important example of a multidimensional integrable system, namely the anti-self-dual Einstein equations. These define a 4dimensional (possibly complex) metric of signature $(+,+,+,+$ ) with the Ricci tensor zero and the Weyl tensor anti-self-dual [5]. Such metrics have an existence independent of the particular coordinate system used to describe it, and so one of the problems in finding a anti-selfdual Einstein hierarchy is to find a suitable coordinate system in which the field equations take a form similar to equation (1)). For example, all such metrics are automatically Kähler [6], and so the metric may be written in terms of a single scalar function, the Kähler potential. The field equation is then

$$
\Omega_{, w y} \Omega_{, x z}-\Omega_{, w z} \Omega_{, x y}=1
$$

(and is known as Plebañski's first heavenly equation [7]) and in this form it is not at all obvious what the recursion operator should be, nor how to construct the associated hierarchy.

One possible way forward is via the use of the infinite dimensional Lie algebra $\operatorname{sdiff}\left(\Sigma^{2}\right)$ of area-preserving diffeomorphism of a 2-surface $\Sigma^{2}$, where one replaces the Lie bracket $[A, B]=A B-B A$ of matrices by the Poisson bracket of functions

$$
\{A, B\}=\frac{\partial A}{\partial y} \frac{\partial B}{\partial z}-\frac{\partial A}{\partial z} \frac{\partial B}{\partial y} .
$$

Park [8] has shown that one may interpret (22) as a 2-dimensional topological chiral model with fields taking values in the Lie algebra $\operatorname{sdiff}\left(\Sigma^{2}\right)$. As this chiral model is a reduction of the anti-self-dual Yang-Mills equations one might hope that by studying an appropriately reduced version of the hierarchy one could obtain a chiral field hierarchy, and then, via the replacement $[A, B] \rightarrow\{A, B\}$ to a antiself-dual Einstein hierarchy. This convoluted approach will be avoided for two reason. Firstly, underlying these anti-self-dual integrable systems are two quite different geometric constructions. Solutions to the anti-self-dual Yang-Mills equations are encoded in certain holomorphic vector bundles over a flat twistor space [9], while solutions to the antiself-dual Einstein equations are encoded within a curved twistor space [5]. Thus replacing matrix commutators by Poisson brackets only works at the level of the differential equations, not in the underlying geometry. Secondly, implicit in much of the work on anti-self-dual Yang-Mills hierarchies is the fact that the zero-curvature relation 


$$
\partial_{x} A-\partial_{y} B+[A, B]=0
$$

may be solved by taking $A=g^{-1} \partial_{y} g, B=g^{-1} \partial_{x} g$, for some groupvalued element $g$. Unfortunately this does not hold under the replacement $[A, B] \rightarrow\{A, B\}$, there being no notion of pure gauge.

\section{The Cauchy-Kovalevski form of the anti- self-dual Einstein equation}

By defining a new independent variable $t=-\Omega_{w}$ (so that now $w=$ $w(t, x, y, z))$ and performing a Legendre transformation [10] with

$$
h(t, x, y, z)=\Omega(w(t, x, y, z), x, y, z)+t w(t, x, y, z),
$$

Plebañski's first heavenly equation (22) takes the new form

$$
h_{t t}=h_{x z} h_{t y}-h_{x y} h_{t z} .
$$

This is in the Cauchy-Kovalevski form, and a formal solution may be obtained as a power series in $t$, depending on the initial data $\left.h\right|_{t=0}$ and $\left.\partial_{t} h\right|_{t=0}$. Equation (4) was first derived by Grant [11], using a different approach which will be given in the next section. The corresponding anti-self-dual Einstein metric is

$$
g=d t\left(h_{t y} d y+h_{t z} d z\right)+d x\left(h_{x y} d y+h_{x z} d z\right)+\frac{1}{h_{t t}}\left(h_{t y} d y+h_{t z} d z\right)^{2} .
$$

To put equation (4) into a form similar to (11) let $u=h_{t}$, so $h_{x}=$ $\partial_{t}^{-1} u_{x}$. With this (4) becomes

$$
\begin{aligned}
u_{t} & =u_{y}\left(\partial_{t}^{-1} u_{x}\right)_{z}-u_{z}\left(\partial_{t}^{-1} u_{x}\right)_{y} \\
& =\left[\left(u_{y} \partial_{z}-u_{z} \partial_{y}\right) \partial_{t}^{-1}\right] u_{x},
\end{aligned}
$$

which, with the help of (3), becomes

$$
u_{t}=\left\{u, \partial_{t}^{-1} u_{x}\right\}
$$

This suggest that $\left\{u, \partial_{t}^{-1}\right\}$ might be a recursion operator. This is indeed the case, as will be shown in the next two sections, and hence the anti-self-dual Einstein hierarchy is given by 


$$
u_{t}=\left\{u, \partial_{t}^{-1}\right\}^{n} u_{x}
$$

Simple solutions to (6) may be found quite easily. With the ansatz

$$
u=-z+\frac{\partial^{n} g(t, x, y)}{\partial t^{n}}
$$

the nonlinearities disappear, and one is left with a linear equation for the function $g(t, x, y)$ :

$$
\frac{\partial^{n+1} g}{\partial t^{n+1}}-\frac{\partial^{n+1} g}{\partial y^{n} \partial x}=0
$$

Solutions to this equation may written in terms of a contour integral

$$
g(t, x, y)=\frac{1}{2 \pi i} \oint k\left(\xi, y+\xi t+\xi^{n+1} x\right) d \xi,
$$

where $k(\xi, \omega)$ is an arbitrary holomorphic function. This extends Bateman's formula for the $n=1$ case, which gives metrics with anti-self-dual Killing vectors [12].

The appearance of the operator $\partial_{t}^{-1}$ in (5) is somewhat unusual. On interchanging $x$ and $t$ one can rewrite this as

$$
u_{t}=\left\{u, \partial_{x}^{-1}\right\}^{-1} u_{x}
$$

so no $t$-derivatives of integrals appear on the right hand side. This, however, is at the cost of introducing the inverse Poisson bracket operator, and so to avoid this the form (5) will be used (recall, if $\mathcal{R}$ is a recursion operator then $\mathcal{R}^{-1}$ is also). Before proving that $\left\{u, \partial_{t}^{-1}\right\}$ is a recursion operator, the Lax pair for the hierarchy (6) will be found.

\section{The Lax Equations for the anti-self-dual Einstein hierarchy}

One of the ways in which the construction of anti-self-dual Einstein metrics may be understood is as follows [13, 14, 15]. Let $\mathcal{L}_{0}=V_{1}+\xi V_{2}$ and $\mathcal{L}_{1}=V_{3}+\xi V_{4}$ be commuting vector fields on some 4-manifold $\mathcal{M}$ for all values of the parameter $\xi$ (the spectral parameter), with each of the $V_{i}$ preserving a four-form $\omega$ on $\mathcal{M}$. With $\Lambda=\omega\left(V_{1}, V_{2}, V_{3}, V_{4}\right)$ one may define a (contravariant) metric 


$$
g=\Lambda^{-1}\left(V_{1} \otimes_{s} V_{4}-V_{2} \otimes_{s} V_{3}\right)
$$

and this metric is automatically an anti-self-dual Einstein metric. Conversely, all such metrics can be constructed in this manner. In what follows it will be convenient to defined a vector field $L_{u}$, depending on some function $u(t, x, y, z)$ by

$$
L_{u}=\frac{\partial u}{\partial y} \frac{\partial}{\partial z}-\frac{\partial u}{\partial z} \frac{\partial}{\partial y} .
$$

One can define the commutator of $L_{u}$ and $L_{v}$ in two different, but equivalent ways:

- Regard $L_{u}$ and $L_{v}$ as differential operators, and define $\left[L_{u}, L_{v}\right]=$ $L_{u} L_{v}-L_{v} L_{u}$,

- Regard $L_{u}$ and $L_{v}$ as vector fields, and define the commutator to be the Lie bracket of vector fields.

In each case one finds $\left[L_{u}, L_{v}\right]=L_{\{u, v\}}$, where $\{u, v\}$ is the Poisson bracket defined by (3).

What Grant [11] did was to take

$$
\begin{aligned}
V_{1} & =\partial_{t}, \\
V_{2} & =-L_{u}, \\
V_{3} & =\partial_{x}, \\
V_{4} & =-\partial_{t}-L_{v}
\end{aligned}
$$

and $\omega=d t \wedge d x \wedge d y \wedge d z$. The condition that the vector fields $\mathcal{L}_{0}$ and $\mathcal{L}_{1}$ commute results in equations

$$
\begin{aligned}
& u_{t}=\{u, v\} \\
& v_{t}=u_{x},
\end{aligned}
$$

and on eliminating $v$ one obtains equation (四). An equivalent pair of vector fields, now no longer linear in the constant $\xi$ are

$$
\begin{aligned}
& \mathcal{L}_{0}=\partial_{t}-\xi L_{u}, \\
& \mathcal{L}_{1}=\partial_{x}-\xi L_{v}-\xi^{2} L_{u},
\end{aligned}
$$


and for these to commute equation (1) has to hold. Motivated by the structure of the AKNS hierarchy, one can propose that the anti-selfdual Einstein hierarchy be given by solving the integrability conditions for the two vector fields

$$
\begin{aligned}
\mathcal{L}_{0} & =\partial_{t}-\xi L_{u}, \\
\mathcal{L}_{1} & =\partial_{x}-\sum_{r=1}^{n} \xi^{r} L_{v_{r}} .
\end{aligned}
$$

The coefficient of the heighest power of $\xi$ in the commutator $\left[\mathcal{L}_{0}, \mathcal{L}_{1}\right]$ is $L_{\left\{v_{n}, u\right\}}$, and for this to be zero one may take $v_{n}=u$ (in general, one has $v_{n}=\alpha u+\beta(t, x)$ but this may be converted to the above form by various redefinition of the variables). Using this result one finds, on equating the various powers of $\xi$, that

$$
\begin{aligned}
u_{t} & =\left\{u, v_{n-1}\right\}, \\
v_{i, t} & =\left\{u, v_{i-1}\right\}, \quad i=2, \ldots, n-1, \\
v_{1, t} & =u_{x} .
\end{aligned}
$$

This system is also in Cauchy-Kovalevski form, and so formal solutions may be obtained as power series in $t$. Systematically eliminating the $v_{i}$ in favour of the function $u$ results in the single equation

$$
\begin{aligned}
u_{t} & =\left\{u, \partial_{t}^{-1}\left\{u, \partial_{t}^{-1}\left\{\ldots \partial_{t}^{-1}\left\{u, \partial_{t}^{-1}, u_{x}\right\} \ldots\right\}\right\}\right\} \\
& =\left\{u, \partial_{t}^{-1}\right\}^{n} u_{x}
\end{aligned}
$$

Thus the operator $\left\{u, \partial_{t}^{-1}\right\}$ acts as a recursion operator. In the next section the symmetry properties of this operator will be considered.

In the above one has a single scalar field $u(t, x, y, z)$. One may extend this to $m$-scalar fields by considering the vector fields

$$
\begin{aligned}
& \mathcal{L}_{0}=\partial_{t}-\sum_{r=1}^{m} \xi^{r} L_{u_{r}}, \\
& \mathcal{L}_{1}=\partial_{t}-\sum_{s=1}^{n} \xi^{s} L_{v_{s}} .
\end{aligned}
$$


Without loss of generality one may take $n \geq m$ (otherwise just interchange $x$ and $t$ ) and the integrability condition $\left[\mathcal{L}_{0}, \mathcal{L}_{1}\right]=0$ can be solved at each power of $\xi$, expressing the $v_{i}$ in terms of the $u_{i}$. This results in the system of equations for the $m$-scalar fields

$$
\left(\begin{array}{c}
u_{1} \\
\vdots \\
u_{m}
\end{array}\right)_{t}=\mathcal{R}_{m}^{n-m}\left(\begin{array}{c}
u_{1} \\
\vdots \\
u_{m}
\end{array}\right)_{x}
$$

where $\mathcal{R}_{m}$ is the matrix recursion operator

$$
\mathcal{R}_{m}=\left(\begin{array}{ccccccc}
\left\{u_{1}\right. & , \partial_{t}^{-1} & \} & 1 & 0 & \ldots & 0 \\
\left\{u_{2}, \partial_{t}^{-1}\right. & \} & 0 & 1 & \ldots & 0 \\
\vdots & & \vdots & \vdots & \ddots & \vdots \\
\left\{u_{m-1}, \partial_{t}^{-1}\right. & \} & 0 & 0 & \ldots & 1 \\
\left\{u_{m}\right. & , \partial_{t}^{-1} & \} & 0 & 0 & \ldots & 0
\end{array}\right)
$$

Once again, the associated symmetry properties of this generalised hierarchy will be considered in the next section.

\section{Symmetries of the anti-self-dual Ein- stein hierarchy}

As mentioned in the introduction, one of the ways in which the recursion operator may be introduced is via the study of the symmetries of the original equation (see [16] and [17]). Given an evolution equation of the form

$$
u_{t}=K(u)
$$

a hidden symmetry $\tau$ corresponds to an infinitesimal parameter satisfying

$$
\tau_{t}=K^{\prime}[\tau]
$$

where $K^{\prime}[\tau]$ is the Fréchet derivative of $K(u)$. Given an operator $\mathcal{R}$ satisfying the equation

$$
\mathcal{R}^{\prime}[K]=\left[K^{\prime}, \mathcal{R}\right]
$$


one may generate a hierarchy of symmetries, since

$$
\begin{aligned}
(\mathcal{R} \tau)_{t} & =\mathcal{R}_{t} \tau+\mathcal{R} \tau_{t} \\
& =\mathcal{R}^{\prime}[K] \tau+\mathcal{R} K^{\prime}[\tau] \\
& =K^{\prime}[\mathcal{R} \tau]
\end{aligned}
$$

Such an operator is said to be a strong symmetry, or recursion operator. For $\mathcal{R}$ to be a strong symmetry for the hierarchy

$$
u_{t}=\mathcal{R}^{n} u_{x}
$$

it has to be a hereditary (or Nijenhuis) operator:

$$
\left(\mathcal{R}^{\prime}[\mathcal{R} f] g-\mathcal{R} \mathcal{R}^{\prime}[f] g\right)-\left(\mathcal{R}^{\prime}[\mathcal{R} g] f-\mathcal{R} \mathcal{R}^{\prime}[g] f\right)=0
$$

In this section it will be shown that $\mathcal{R}_{m}$, given by (8) is both a strong symmetry and a hereditary operator. The proof will follow [18] where the symmetries of a 2-dimensional chiral model were studied.

The field equations (17) are

$$
u_{i, t}=K(\mathbf{u})_{i}
$$

where

$$
K(\mathbf{u})_{i}= \begin{cases}\left\{u_{i}, \partial_{t}^{-1} u_{1, x}\right\}+u_{i+1, x} & i=1, \ldots, m-1 \\ \left\{u_{m}, \partial_{t}^{-1} u_{1, x}\right\} & i=m\end{cases}
$$

and the conjectured recursion operator is

$$
(\mathcal{R} \mathbf{f})_{i}= \begin{cases}\left\{u_{i}, \partial_{t}^{-1} f_{1}\right\}+f_{i+1} & i=1, \ldots, m-1 \\ \left\{u_{m}, \partial_{t}^{-1} u_{1}\right\} & i=m\end{cases}
$$

The relevant Fréchet derivatives are

$$
K^{\prime}[\mathbf{f}]_{i}= \begin{cases}\left\{f_{i}, \partial_{t}^{-1} u_{1, x}\right\}+\left\{u_{i}, \partial_{t}^{-1} f_{1, x}\right\}+f_{i+1, x} & i=1, \ldots, m-1 \\ \left\{f_{m}, \partial_{t}^{-1} u_{1, x}\right\}+\left\{u_{n}, \partial_{t}^{-1} f_{1, x}\right\} & i=m,\end{cases}
$$

and 


$$
(\mathcal{R}[\mathbf{g}] \mathbf{f})_{i}=\left\{g_{i}, \partial_{t}^{-1} f_{1}\right\} \quad i=1, \ldots, m
$$

These may be simplified by assuming $f_{i}=0$ and $u_{i}=0$ for $i>m$.

- $\mathcal{R}$ is a strong symmetry.

For arbitrary $m$-component vectors $\mathbf{f}$ and $\mathbf{g}$ one has, by direct calculation,

$$
\begin{aligned}
\left(\mathcal{R}^{\prime}[K] \mathbf{f}\right)_{i}= & \left\{\left\{u_{i}, \partial_{t}^{-1} u_{1, x}\right\}+u_{i+1, x}, \partial_{t}^{-1} f_{1}\right\} \\
\left(\mathcal{R} K^{\prime}[\mathbf{f}]\right)_{i}= & \left\{u_{i}, \partial_{t}^{-1}\left(\left\{f_{1}, \partial_{t}^{-1} u_{1, x}\right\}+\left\{u_{1}, \partial_{t}^{-1} f_{1, x}\right\}+f_{2, x}\right)\right\}+ \\
& \left\{f_{i+1}, \partial_{t}^{-1} u_{1, x}\right\}+\left\{u_{i+1}, \partial_{t}^{-1} f_{1, x}\right\}+f_{i+2, x} \\
\left(K^{\prime}[\mathcal{R} \mathbf{f}]\right)_{i}= & \left\{\left\{u_{i}, \partial_{t}^{-1} f_{1}\right\}+f_{i+1}, \partial_{t}^{-1} u_{1, x}\right\}+\left\{u_{i}, \partial_{t}^{-1}\left\{u_{1}, \partial_{t}^{-1} f_{1}\right\}_{x}\right\}+ \\
& \left\{u_{i+1}, \partial_{t}^{-1} f_{1}\right\}_{x}+\left\{u_{i}, \partial_{t}^{-1} f_{2}\right\}_{x}+f_{i+2, x}
\end{aligned}
$$

By judicious use of the Jacobi identity, followed by an integration by parts, it is straightforward to show that

$$
\mathcal{R}^{\prime}[K] \mathbf{f}-K^{\prime}[\mathcal{R} \mathbf{f}]+\mathcal{R} K^{\prime}[\mathbf{f}]=0
$$

and hence $\mathcal{R}$ is a strong symmetry, or a recursion operator.

- $\mathcal{R}$ is an hereditary operator.

Similarly

$$
\begin{aligned}
\left(\mathcal{R}^{\prime}[\mathcal{R} \mathbf{f}] \mathbf{g}\right)_{i} & =\left\{\left\{u_{i}, \partial_{t}^{-1} f_{1}\right\}+f_{i+1}, \partial_{t}^{-1} g_{1}\right\} \\
\left(\mathcal{R R}^{\prime}[\mathbf{f}] \mathbf{g}\right)_{i} & =\left\{u_{i}, \partial_{t}^{-1}\left\{f_{1}, \partial_{t}^{-1} g_{1}\right\}\right\}+\left\{f_{i+1}, \partial_{t}^{-1} g_{1}\right\}
\end{aligned}
$$

and hence (again using the Jacobi identity followed by an integration by parts)

$$
\left(\mathcal{R}^{\prime}[\mathcal{R} \mathbf{f}] \mathbf{g}-\mathcal{R} \mathcal{R}^{\prime}[\mathbf{f}] \mathbf{g}\right)-\left(\mathcal{R}^{\prime}[\mathcal{R} \mathbf{g}] \mathbf{f}-\mathcal{R} \mathcal{R}^{\prime}[\mathbf{g}] \mathbf{f}\right)=0
$$

for arbitrary $\mathbf{f}$ and $\mathbf{g}$, thus showing $\mathcal{R}$ is a hereditary operator. 
Thus given any symmetry $\tau$ one may construct a hierarchy of related symmetries by the repeated application of $\mathcal{R}$. It thus remains to find some initial symmetries to start off this procedure, and to find the Lie algebra generated by these symmetries. For simplicity only the $m=1$ case will be considered, i.e. the anti-self-dual Einstein hierarchy itself and not the more general system given by (7).

Two simple solutions of equation (9) are given by

$$
\begin{aligned}
\tau_{p}^{(0)} & =\{u, p(y, z)\} \\
\tau^{(0)} & =\mathcal{R} u
\end{aligned}
$$

(note that although $\mathcal{R} u$ is a symmetry, $u$ is not, since $2 u_{t}=K^{\prime}[u]$ ). Thus, by the action of $\mathcal{R}$, one has the following hierarchies of symmetries:

$$
\left.\begin{array}{rl}
\tau_{p}^{(n)} & =\mathcal{R}^{n} \tau_{p}^{(0)} \\
\tau^{(n)} & =\mathcal{R}^{n} \tau^{(0)}
\end{array}\right\} n=0,1,2, \ldots
$$

Following the argument in 18 (which makes use of the hereditary property of $\mathcal{R}$ ) one may easily show that the Lie algebra of these symmetries is given by

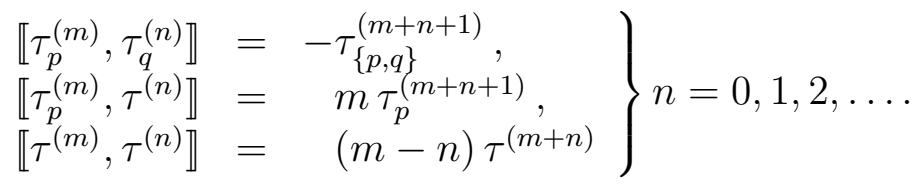

where $p$ and $q$ are functions of $y$ and $z$ alone, and the Lie bracket $\llbracket F, G \rrbracket$ is defined by

$$
\llbracket F, G \rrbracket=F^{\prime}[G]-G^{\prime}[F]
$$

Similar results have been obtained by Grant [11], who has calculated the Lie-point symmetries of equation (4). The Lie point symmetries of Plebañski's equation (2) has been calculated by Boyer and Winternitz [19], and it would be interesting to see how the Legendre transformation that takes (4) to (2) connects the two sets of symmetries.

Note that the recursion operator $\mathcal{R}$ factors,

$$
\mathcal{R}=\mathcal{H}_{1} \mathcal{H}_{0}^{-1}
$$

where 


$$
\begin{aligned}
\mathcal{H}_{0} & =\partial_{t}, \\
\mathcal{H}_{1} & =\{u, \quad\} .
\end{aligned}
$$

This suggests a bi-Hamiltonian structure for the anti-self-dual Einstein hierarchy. However one would need to study the corresponding Hamiltonians in greater detail to verify this. The same splitting holds for the more general hierarchy given by equations (7) and (8).

\section{Comments}

In this paper the symmetry structure of the anti-self-dual Einstein equations have been investigated. This work raises a number of further question, mainly connected to the geometry of both the equations and its symmetries. The dressing properties of the Lax pair for the self-dual Einstein equations (in Plebañski's form) and its reductions has been extensively studied by Takasaki and Takebe [20], who has also found the $\tau$-function for the system. It therefore seems important to generalize these ideas to the hierarchy considered here, and to understand the action of the symmetries in terms of the dressing operation.

If the Poisson bracket $\{A, B\}$ were to be replaced by the matrix commutator $[A, B]$ (with the now matrix valued fields being functions of $x$ and $t$ alone) then the results of this paper will still hold, the basic equations being those of a Wess-Zumino-Witten topological field theory. Thus one obtains a topological field theory hierarchy together with its symmetries. In this case the geometry is much easier to understand, as the equations are a reduction of the generalised self-dual Yang-Mills equations introduced in [2]. Here the (flat) twistor space $T \cong \mathcal{O}(1) \oplus \mathcal{O}(1)$ (this being $C P^{3}$ with a single projective line removed) is replaced by $T_{m, n} \cong \mathcal{O}(m) \oplus \mathcal{O}(n)$ and the Ward construction carries over naturally to this case. For the integrable systems under consideration here what one needs is an analogous generalization of Penrose's non-linear graviton construction [5]. One possibility is to consider curved twistor spaces $\mathcal{T}_{m, n}$ with normal bundle $\mathcal{O}(m) \oplus \mathcal{O}(n)$. Such spaces have been considered by Gindikin [21], and it remains to see how the integrable systems studied here fit into this framework.

One may also construct a 3-dimensional hierarchy by assuming that the Kähler potential has the form $\Omega=\Omega(\log w+\log x, y, z)$ and a Legendre transformation takes (2) to 


$$
\nabla^{2} u+\frac{\partial^{2} e^{u}}{\partial t^{2}}=0
$$

(the so-called $S U(\infty)$-Toda, or Boyer-Finley equation) or to

$$
h_{t t}=e^{-v}\left(h_{v s} h_{v t}-h_{v v} h_{s t}\right)
$$

(where $v=\log w+\log x$ ). One may perform a similar reduction to the hierarchy (6), the lowest member of which is given by (10). It would be interesting to see if this hierarchy corresponds to the $S U(\infty)$-Toda hierarchy constructed and studied by Takasaki and Takebe 20.

The symmetries of the full (i.e. with no restriction on the Weyl tensor) Einstein equations have been studied recently by Torre and Anderson [22], who have found that no non-trivial generalised symmetries

exist. Their approach is coordinate independent, and it might be of interest to apply these method to the anti-self-dual case.

It is hoped that some of these issues will be addressed in a future paper.

\section{Acknowledgements}

I would like to thank James Grant for a number of useful conversations, and also the University of Newcastle for a Wilfred Hall fellowship.

\section{References}

[1] Ward, R.S., Nucl. Phys. B236 (1984) 318-396.

[2] Strachan, I.A.B., J. Math. Phys. 34 (1993) 243-259.

[3] Ablowitz, M.J., Chakravarty, S. and Takhtajan, L., Commun. Math. Phys. 158 (1994) 289-314.

[4] Schiff, J., Self-dual Yang-Mills and the Hamiltonian structures of integrable systems, preprint IASSNS-HEP-92/34, hep-th/9211070.

[5] Penrose, R. Gen. Rel. Grav. 7 (1976) 31-52. 
[6] Atiyah, M.F., Hitchin, N.J. and Singer, I.M., Proc. Roy. Soc. Lond. A362 (1978) 425-461.

[7] Plebañski, J.F., J. Math. Phys. 16 (1975) 2395-2402.

[8] Park, Q-Han., Int. J. Mod. Phys. A7 (1992) 1415-1448.

[9] Ward, R.S., Phys. Lett. 61A (1977) 81-82.

[10] Finley III, J.D., Plebañski, J.F., Przanowski, M. and GarciaCompean H. Phys. Lett. 181A (1993) 434-442.

[11] Grant J., Phys. Rev. D48 (1993) 2606-12.

[12] Tod, K.P. and Ward, R.S., Proc. R. Soc. A386 (1979) 411-427.

[13] Ashtekar, A., Jacobson, T. and Smolin, L., Commun. Math. Phys. 115 (1988) 631-648.

[14] Mason, L.J. and Newman, E.T., Commun. Math. Phys. 121 (1989) 659-668.

[15] Ward, R.S., Class. Quantum Grav. 7 (1990) L217-L222.

[16] Magri, F., in Lecture Notes in Physics vol.120 (1980) ed. Boiti, M. et. al. (Springer, Berlin).

[17] Fuchssteiner, B. and Fokas, A.S., Physica 4D 47-66.

[18] Cheng, Y., J. Phys. A 21 (1988) L715-L718.

[19] Boyer, C.P. and Winternitz, P., J. Math. Phys. 30 (1989) 10811094.

[20] Takasaki, K. and Takebe, T., Lett. Math. Phys. 23 (1991) 205-214.

[21] Gindikin, S.G., in Twistors in Mathematics and Physics ed. Bailey, T.N. and Baston, R.J. (C.U.P., Cambridge).

[22] Anderson, I.M. and Torre, C.G., Classification of generalized symmetries for the vacuum Einstein equations, preprint, Utah 1994, gr-qc/9404030. 